\title{
Near-field Radiative Thermal Modulation by Tunneling Through Graphene Sheet
}

\author{
Yizhi Hu, Xianglin Tang, Yonggang Zhu and Yue Yang*
}

\begin{abstract}
A bstract
In this paper, we have theoretically demonstrated a graphene-mediated near-field radiative thermal modulator based on doped silicon-graphene-doped silicon three-slab configuration. The near-field photon tunneling between the doped silicon emitter and receiver is modulated by changing chemical potential of graphene sheet and the separation distance between the sheet and the emitter. The near-field three-body theory built on fluctuational electrodynamics is used to calculate total radiative heat flux, which could be modulated in a range of $10-70 \mathrm{~kW} / \mathrm{m}^{2}$ with different setup for graphene chemical potential and its position. The underlying mechanism is illustrated as varied coupling behavior of surface plasmon polaritons between doped silicon and graphene sheet. Several dimensionless factors such as normalized heat flux, sensitivity factor and switching factor are also introduced for comprehensive analysis of the performance of modulation effect. The results obtained here will trigger a new way for near-field active thermal management between bulk materials utilizing suspended 2D materials.
\end{abstract}

Keywords: near field radiation; thermal modulation; three-slab configuration; graphene sheet; surface plasmon polaritons Received: 3 February 2020; Accepted: 4 March 2020

Article type: Research article

\section{Introduction}

For classic thermal radiation, the radiative heat flux between two bodies is governed by propagation waves which obey the Stefan-Boltzmann law. If the distance is smaller than the characteristic wavelength of thermal radiation predicted by Wien 's displacement law, the interference of evanescent waves and photon tunneling would contribute to dramatic increase in radiative heat transfer, which is known as nearfield thermal radiation. ${ }^{[1,2]}$ Moreover, in near-field regime, heat flux will show a further enhancement due to the excitation of additional resonance modes, such as surface plasmon polaritons (SPPs), ${ }^{[3-5]}$ surface phonon polaritons ${ }^{[6-8]}$ and magnetic polaritons. ${ }^{[9,10]}$

Recently, considering practical application scenarios, how to regulate near-field heat flux has become one of hot research topics. ${ }^{[11]}$ A variety of near-field radiative thermal transistor, ${ }^{[12,13]}$ rectifier, ${ }^{[14-19]}$ switch $^{[20,21]}$ and thermostat ${ }^{[22]}$ have been theoreti-

School of Mechanical Engineering and Automation, Harbin Institute of Technology, Shenzhen, 518055, PR China

*E-mail: yangyue2017@hit.edu.cn (Y. Yang) cally designed and studied to actively control heat flux utilizing different mechanisms. Adding another body between two fixed bodies will achieve a notable change in primary radiative heat flux, which already becomes a novel way to adjust near-field photon transport. ${ }^{[23-25]}$ Ben-Abdallah et al. ${ }^{[12]}$ designed a near-field thermal transistor based on such a threeparallel-slabs system and the Mott phase transition of intermediate vanadium dioxide $\left(\mathrm{VO}_{2}\right)$ slab, which can realize the functions of thermal switch, amplification and modulation simultaneously. A thermal rectification was also proposed by Gu et al. ${ }^{[2]]}$ which could mediate near-field thermal transfer via the control of the separation distance between the central $\mathrm{VO}_{2}$ part and the emitter part. The key of these configurations is the use of insulator-metal transition material, ${ }^{[26,2]}$ which is capable of changing its own optical property through temperature variation, resulting in coupling or mismatch of surface resonant modes. Thus, the near-field heat flux will vary accordingly. However, the optical characteristic change of insulator-metal transition material is so strongly dependent on threshold temperature that the regulation speed, resolution, and 
flexibility are limited.

Graphene, a two-dimensional material, has attracted extensive attentions because of its unique and astonishing electronic and optical properties. ${ }^{[28]}$ One of the main features is tunable surface plasmon ${ }^{[29,30]}$ with variable chemical potentials induced by external voltage biases or doping. The feature makes graphene become a favorable alternative for radiative thermal management device. ${ }^{[31-34]}$ Yang et al. ${ }^{[3]}$ conceived of an electrically-controlled near-field thermal modulator made of two graphene-coated silicon carbide plates. He et al. ${ }^{[2]}$ designed a near-field thermostat consisted of graphene-coated dielectric emitter, receiver and intermediate layer without graphene. Similarly, changing chemical potentials of graphene by gate voltage, the temperature of intermediate body can be reset due to the coupling of graphene plasmon and dielectric phonon polaritons.

In this paper, a two-parameter near-field radiative thermal modulator made of three-slab system is introduced. The system basically has semi-infinite doped silicon slab emitter and receiver. In the middle of them, a monolayer of graphene is placed in parallel to make near-field radiative photons tunnel through. It is similar to other graphene-based modulator, and opto-electronic control technology is adopted to tune the chemical potentials of graphene, which possesses a superiority of fast operating speed and high resolution. Moreover, the distance between emitter and graphene sheet is also tunable to guarantee regulative magnitude at a larger scale and achieve a better modulating performance. Based on such a twoparameter mediated three-slab configuration, the total radiative heat flux can be varied in a range of $10-70 \mathrm{~kW} / \mathrm{m}^{2}$ for the coupling of graphene plasmon and doped silicon plasmon polaritons. Although theoretically calculated results provide us more flexibility for heat flux modulation with three-body configuration, we also need to notice that it will be more challenging to implement experimental measurement than that with two-body system. A possible way we can think about to achieve it is by dividing it into two separate two-body systems. Through fixing the middle layer position, we can approach the other two bodies to it within a small gap distance by using the existing methods like custom-built nano-positioning method ${ }^{[36]}$ and piezoelectric motor technology. ${ }^{[37]}$ Thermal modulation and switching effects, which are indispensable features of modulator, are quantitatively studied with the independent or simultaneous change of two parameters in detail.

\section{Physical model and theoretical calculation}

\subsection{Physical model}

The configuration we study consists of three separate parallel slabs labeled with subscripts 1-3, which is schematically shown in Fig. 1. The slabs 1 and 3 located on both sides of the configuration are set as doped $\mathrm{Si}$ and they are supposed as semi-infinite. A classic Drude model is used to characterize the dielectric function of doped $\mathrm{Si}^{[[5]}$ (a)

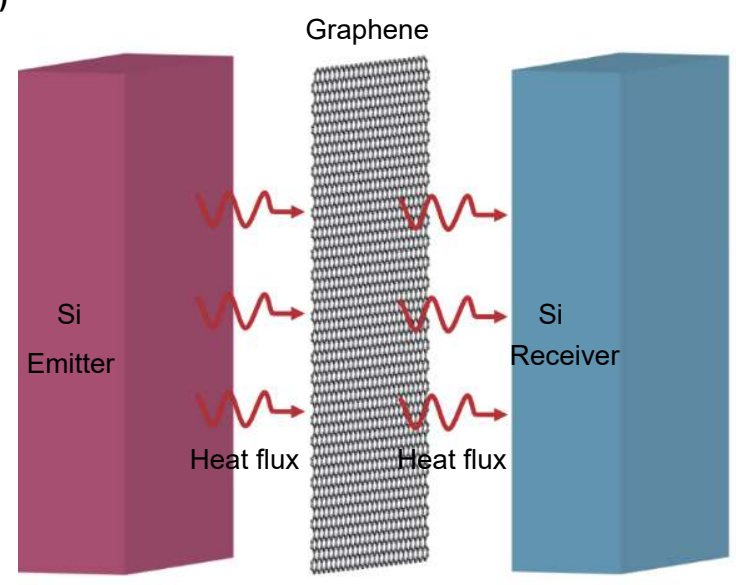

(b)

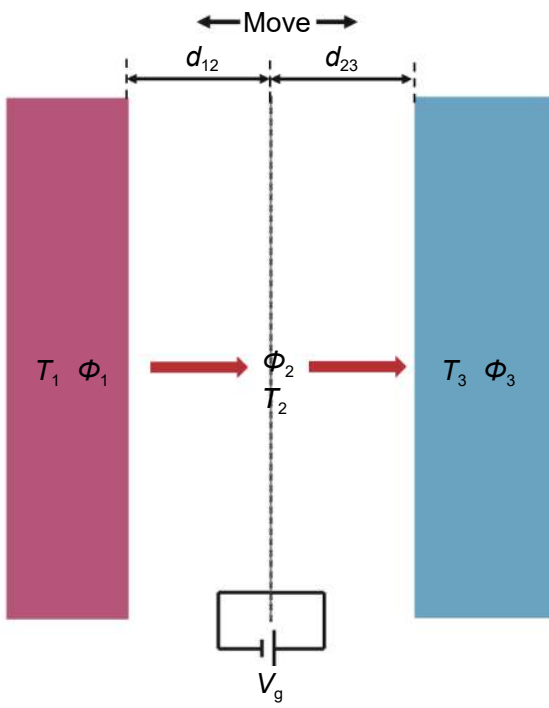

Fig. 1 (a) Schematic illustration of proposed near-field radiative thermal modulator made of Si-graphene-Si three-slab configuration. The three separated slabs are labeled 1-3 and the temperatures of Si emitter and receiver are set as $T_{1}=400 \mathrm{~K}$ and $T_{3}=300 \mathrm{~K}$. The slabs on two sides of the configuration are semi-infinite. The intermediate slab is graphene sheet with thickness $t_{\mathrm{g}}$ and temperature $T_{2}$. (b) Side view of the configuration. The heat flux emitted by slab 1 and obtained by slab 3 are $\Phi_{1}$ and $\Phi_{3}$, respectively. The separation distance between slab 1 and graphene slab is $d_{12}$, while the distance between graphene and slab 2 is $d_{23}$ and $d_{12}+d_{23}=100 \mathrm{~nm}$. The position of graphene slab can be moved, while the chemical potentials of it can also be changed.

$$
\varepsilon_{\mathrm{Si}}(\omega)=\varepsilon_{\infty}-\frac{\omega_{\mathrm{p}}^{2}}{\omega^{2}+i \gamma \omega},
$$

where $\varepsilon_{\infty}=11.7, \quad \omega_{\mathrm{p}}=1.08 \times 10^{15} \mathrm{rad} / \mathrm{s}$ is the plasma frequency and the scattering rate $\gamma$ is taken as $9.34 \times 10^{13}$ $\mathrm{rad} / \mathrm{s}$, which are corresponding to a doping concentration on the order of $10^{20} \mathrm{~cm}^{-3}$ at room temperature. The reason to 
choose this doping concentration is to maintain Si SPPs within the frequency domain that Graphene SPPs can match. The slab 1 is hot emitter and slab 3 is cold receiver, which are kept unchanged at $T_{1}=400$ and $T_{3}=300 \mathrm{~K}$, respectively. The thin slab 2 is a monolayer graphene with thickness $t_{\mathrm{g}}=0.3 \mathrm{~nm}$, which is placed between slab 1 and slab 3 at a distance of $d_{12}$ away from slab 1 and $d_{23}$ from slab 3 . It should be noted that the $d_{12}$ and $d_{23}$ are not independent and constrained by $d_{12}+d_{23}=100 \mathrm{~nm}$. The graphene layer can be moved via the piezoelectric motor, inducing the changes in $d_{12}$ and $d_{23}$, and the corresponding heat flux will get modulated. In our present work, the graphene sheet is modeled as a medium with dielectric function determined by the Drude model: ${ }^{[38]}$

$$
\begin{gathered}
\varepsilon_{\mathrm{g}}(\omega)=1+\frac{\mathrm{i} \sigma_{\mathrm{g}}}{\omega \varepsilon_{0} t_{\mathrm{g}}}, \\
\sigma_{\mathrm{g}}=\sigma_{\mathrm{d}}+\sigma_{\mathrm{i}}, \\
\sigma_{\mathrm{d}}=\frac{\mathrm{i}}{\omega+\mathrm{i} / \tau} \frac{2 e^{2} k_{\mathrm{B}} T_{2}}{\pi \hbar^{2}} \ln \left[2 \cosh \left(\frac{\mu}{2 k_{\mathrm{B}} T_{2}}\right)\right] \\
\sigma_{\mathrm{i}}=\frac{e^{2}}{4 \hbar}\left[G\left(\frac{\hbar \omega}{2}\right)+\mathrm{i} \frac{4 \hbar \omega}{\pi} \int_{0}^{\infty} \frac{G(\xi)-G(\hbar \omega / 2)}{(\hbar \omega)^{2}-4 \xi^{2}} \mathrm{~d} \xi\right]
\end{gathered}
$$

where $\varepsilon_{0}$ is the permittivity in vacuum and $\sigma_{\mathrm{g}}$ indicates the conductivity of graphene, which is a sum of an intraband term $\sigma_{\mathrm{d}}$ and an interband term. Note that $\sigma_{\mathrm{d}}$ stands for devotion from the intraband electron-photon scattering, while $\sigma_{\mathrm{i}}$ is related to the interband electron $\operatorname{draft} . G(\xi)$ is defined as $\sinh \left(\frac{\xi}{k_{\mathrm{B}} T_{2}}\right) /\left[\cosh \left(\frac{\mu}{k_{\mathrm{B}} T_{2}}\right)+\cosh \left(\frac{\xi}{k_{\mathrm{B}} T_{2}}\right)\right]$, relaxation time $\tau$ is chosen as $10^{-13} \mathrm{~s}$ at room temperature, ${ }^{[3]} e$ is the magnitude of electron charge, $\hbar$ is the reduced Planck's constant, $k_{\mathrm{B}}$ is the Boltzmann constant and $\mu$ is chemical potential, which can be tuned by applying voltage bias and thereby change the dielectric property as well as radiative heat flux. Although it is not shown here, we have plotted $\sigma_{\mathrm{g}}$ with different chemical potentials at both $300 \mathrm{~K}$ and $400 \mathrm{~K}$, and the consistent values within infrared region indicate the insensitivity to temperature of $\sigma_{\mathrm{g}}$, which could also be verified by the equation applied in Ref. [39-41] to calculate $\sigma_{\mathrm{g}}$ in infrared region. For sake of improving the computational efficiency, we assume that $\sigma_{\mathrm{g}}$ is independent of $T_{2}$ for all the calculations. Another way to calculate the radiative heat transfer contribution of graphene is to construct the Fresnel reflection coefficient at the interface between vacuum and graphene layer directly, which has been proved to be equivalent to our method. ${ }^{[42]}$

\subsection{Theoretical calculation}

Based on fluctuational electrodynamics associated with the stochastic thermal sources, ${ }^{[43]}$ the general analytic expression of radiative heat flux of arbitrary three-body system with three distinct temperatures was theoretically put forward. ${ }^{[2,4]}$ By means of computing the time-averaged Poynting vector, the total heat flux gained by receiver in our configuration is given by the following Landauer-like formula: ${ }^{[12]}$

$$
\begin{aligned}
\Phi_{3}= & \int_{0}^{\infty} \frac{d \omega}{8 \pi^{3}} \sum_{i=\{\mathbf{s}, \mathbf{p}\}} \int \mathrm{d}^{2} \mathbf{k}\left[\Theta_{12}(\omega) \phi_{i}^{12}\left(\omega, \mathbf{k} ; \mu, d_{12}, T_{2}\right)+\right. \\
& \left.\Theta_{23}(\omega) \phi_{i}^{23}\left(\omega, \mathbf{k} ; \mu, d_{23}, T_{2}\right)\right]
\end{aligned}
$$

Here $\phi_{i}^{j k}(\omega, \mathbf{k})$ signifies the transmission factor from slab $j$ to slab $k(j, k \in\{1,2,3\})$, which indicates the tunneling probability of a photon with momentum $\mathbf{k}$ at frequency $\omega$ for all polarization states $i \in\{s, p\}$. It should be noted that, in the infrared region that we are interested in, the transmission factor $\phi_{i}^{j k}(\omega, \mathbf{k})$ is nearly independent of $T_{2}$ due to the insensitivity of the conductivity $\sigma_{\mathrm{g}}$ of intermediate graphene to $T_{2}$ as previously discussed. $\mathbf{k}=\left(k_{x}, k_{y}\right)$ is the wave vector parallel to the slab planes and $\Theta_{j k}(\omega)=\hbar \omega\left(1 /\left[\exp \left(\hbar \omega / k_{B} T_{j}\right)-1\right]-1 /\right.$ $\left.\left[\exp \left(\hbar \omega / k_{B} T_{k}\right)-1\right]\right)$ is the difference of mean energy of two Planck oscillators at frequency $\omega$. According to near-field threebody theory, projecting general scattering operators into a special case, $\phi_{i}^{21}$ and $\phi_{i}^{32}$ can be described as ${ }^{[45]}$

$$
\begin{gathered}
\phi_{i}^{12}(\omega, \mathbf{k})=\left\{\begin{array}{l}
\frac{\left|\tau_{2, i}\right|^{2}\left(1-\left|\rho_{1, i}\right|^{2}\right)\left(1-\left|\rho_{3, i}\right|^{2}\right)}{\left|1-\rho_{12, i} \rho_{3, i} e^{2 j k_{z} d_{23}}\right|^{2}\left|1-\rho_{1, i} \rho_{2, i} e^{2 j k_{z} d_{12}}\right|^{2}}, k<k_{0} \\
\frac{4\left|\tau_{2, i}\right|^{2} \operatorname{Im}\left(\rho_{1, i}\right) \operatorname{Im}\left(\rho_{3, i}\right) e^{-4 \operatorname{Im}\left(k_{z}\right) d_{12}}}{\left|1-\rho_{12, i} \rho_{3, i} e^{2 j k_{z} d_{23}}\right|^{2} \mid 1-\rho_{1, i} \rho_{2, i} e^{\left.2 j k_{z} d_{12}\right|^{2}}}, k>k_{0}
\end{array},\right. \\
\phi_{i}^{23}(\omega, \mathbf{k})=\left\{\begin{array}{l}
\frac{\left(1-\left|\rho_{12, i}\right|^{2}\right)\left(1-\left|\rho_{3, i}\right|^{2}\right)}{\mid 1-\rho_{12, i} \rho_{3, i} e^{\left.2 j k_{z} d_{23}\right|^{2}}, k<k_{0}} \\
4 \operatorname{Im}\left(\rho_{12, i}\right) \operatorname{Im}\left(\rho_{3, j}\right) e^{-2 \operatorname{Im}\left(k_{z}\right) d_{23}}
\end{array}, k>k_{0}\right.
\end{gathered}
$$

for propagating $\left(k<k_{0}\right)$ and evanescent $\left(k>k_{0}\right)$ modes where index $j$ is recorded as imaginary number, $k$ is 2-norm of $\mathbf{k}$ in reciprocal space, $k_{0}=\omega / c$ and $k_{z}=\sqrt{k_{0}^{2}-k^{2}}$ are the wave vector and its tangential component along $\mathrm{z}$ direction in vacuum separately. The Fresnel reflection and transmission coefficients $\rho_{\alpha, i}, \tau_{\alpha, i}(\alpha \in\{1,2,3\})$ for given three slabs dominate the quantity of transmission factor essentially, whose detail form can be found in Ref. [45] and obtained by the transfer-matrix method. Meanwhile, the reflection coefficient corresponding to an ensemble consisting of slab 1 and intermediate slab is given by

$$
\rho_{12, i}=\rho_{2, i}+\left(\tau_{2, i}\right)^{2} \frac{\rho_{1, i} e^{2 j k_{z} d_{12}}}{1-\rho_{1, i} \rho_{2, i} e^{2 j k_{z} d_{12}}} .
$$

It is easy to notice that $\rho_{12, i}$ is influenced by the separation distance $d_{12}$ and chemical potential $\mu$ of graphene sheet. Symmetrically, the heat flux that emitter launches can be written as 


$$
\begin{aligned}
\Phi_{1}= & \int_{0}^{\infty} \frac{d \omega}{8 \pi^{3}} \sum_{i=\{\mathrm{s}, \mathrm{p}\}} \int \mathrm{d}^{2} \mathbf{k}\left[\Theta_{32}(\omega) \phi_{i}^{32}\left(\omega, \mathbf{k} ; \mu, d_{23}, T_{2}\right)+\right. \\
& \left.\Theta_{21}(\omega) \phi_{i}^{21}\left(\omega, \mathbf{k} ; \mu, d_{12}, T_{2}\right)\right]
\end{aligned}
$$

and the transmission factors are updated by exchanging superscripts 1 and 3 trivially.

The operating mode of our modulator is elaborated as follows. Obviously, the heat flux of intermediate slab $\Phi_{2}$ is equal to the difference of $\Phi_{1}$ and $\Phi_{3}$, i.e. $\Phi_{2}=\Phi_{1}-\Phi_{3}$. If there is no variation of interior parameters and external perturbation, the system would tend to the steady state $\Phi_{2}=0$ in case of $T_{1}>T_{3}$. In other words, at the steady state, the amount of cooling at hot emitter is exactly equivalent to the heat flux absorbed by cold receiver (i.e. $\Phi_{1}=\Phi_{3}$ ), which is a quasi-static equilibrium. By taking measures of changing chemical potential $\mu$ or separation distance $d_{12}$ of the intermediate graphene, the total heat flux of system corresponding to the equilibrium state can be modulated. In Fig. 2, the calculated results of varied $\Phi_{1}$ and $\Phi_{3}$ with increasing temperature $T_{2}$ for dissimilar control parameters are shown. The intersection of two branch lines is a pair of physical quantities $\left(T_{2}^{*}, \Phi^{*}\right)$, which implies the equilibrium state. The equilibrium point $\left(T_{2}^{*}, \Phi^{*}\right)$ would change with the variation of chemical potential $\mu$ and distance $d_{12}$. These preliminary results illustrate that total heat flux of such configuration could be well modulated due to the existence of graphene sheet with different $\mu$ and $d_{12}$. In next section, we will display and discuss the detailed regulating results of $\mu$ and $d_{12}$ control along with the corresponding mechanism.

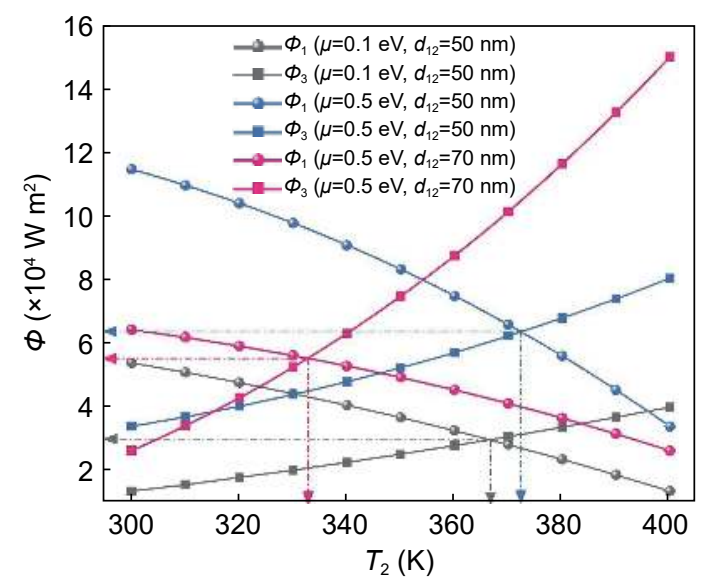

Fig. 2 The lines represent the heat fluxed emitted by slab $1\left(\Phi_{1}\right)$ and heat received by slab $3\left(\Phi_{3}\right)$ with dissimilar cases of chemical potential $\mu$ and separation distance $d_{12}$. The crossover points of $\Phi_{1}$ and $\Phi_{3}$ is pointed out, which infers the equilibrium point $\left(T_{2}^{*}, \Phi^{*}\right)$ of such three-slab system.

\section{Results and discussion}

Now let us introduce the major modulation effects of heat flow from this three-slab configuration. The total radiative flux $\Phi\left(\mu, d_{12}\right)$ of this modulator for equilibrium state is obtained after integration with respect to frequency $\omega$ from 0.1 to
$5 \times 10^{14} \mathrm{rad} / \mathrm{s}$. Please note that $\Phi$ here represents either $\Phi_{1}$ or $\Phi_{3}$ because based on the above analysis, $\Phi_{1}$ is equal to $\Phi_{3}$ at equilibrium state. The frequency range of integration is selected to cover the spectrum of both doped Si and graphene SPPs, and the spectral heat flux out of the range is negligible compared to the part within integration range. For the sake of quantifying the thermal modulation effect of $\mu$ and $d_{12}$ with regard to total heat flux $\Phi\left(\mu, d_{12}\right)$, a dimensionless number (normalized radiative heat flux) is defined as $\Omega=\Phi\left(\mu, d_{12}\right) / \Phi_{0}$, where $\Phi_{0}=26.5 \mathrm{~kW} / \mathrm{m}^{2}$ is the benchmark total radiative heat flux with $\mu=0 \mathrm{eV}$ and $d_{12}=50 \mathrm{~nm}$. Independent regulatory role is taken into consideration from the start, i.e. only one of $\mu$ and $d_{12}$ changes. Instead of total heat flux, the normalized radiative heat flux $\Omega$ as a function of $\mu$ and $d_{12}$ is presented in Fig. 3(a) and 3(b), respectively. Additionally, the variation of equilibrium temperature $T_{2}^{*}$ with $\mu$ or $d_{12}$ is reported together. When $d_{12}$ is fixed at $50 \mathrm{~nm}$, the normalized heat flux $\Omega$ monotonically increases from minimum $\left(\Omega_{\min }=1\right)$ to the maximum $\left(\Omega_{\max }=2.4\right)$ around $\mu=0.5 \mathrm{eV}$, and then decreases slowly with increasing $\mu$. In addition, a similar trend for heat flux change could be observed for the case of $d_{12}=70 \mathrm{~nm}$, where the maximum heat flux exists at $\mu=0.5 \mathrm{eV}$ as well due to strong coupling between $\mathrm{Si}$
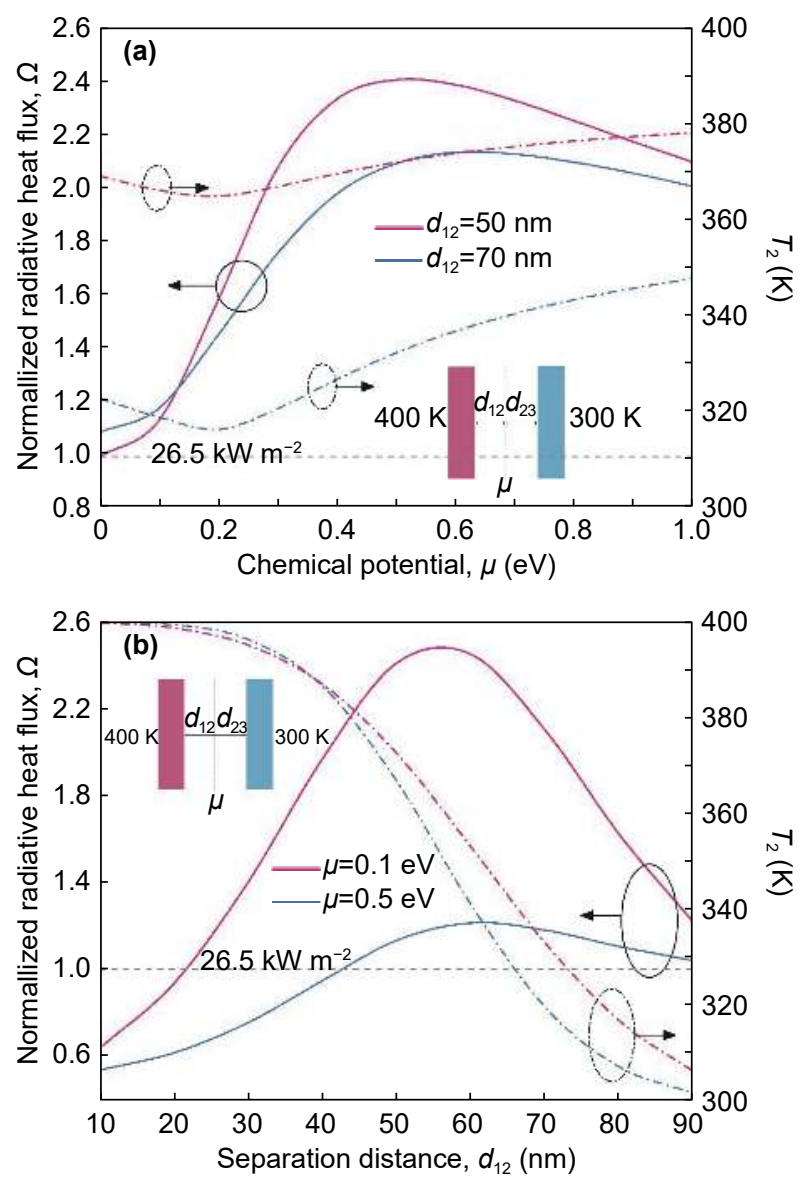

Fig. 3 Normalized radiative heat flux $\Omega$ of such a three-slab configuration as a function of (a) chemical potential $\mu$ when $d_{12}=50$ or $70 \mathrm{~nm}$ and (b) separation distance $d_{12}$ when $\mu=0.1$ or $0.5 \mathrm{eV}$. The corresponding equilibrium temperature $T_{2}$ is also plotted by dashdotted line. 
and graphene SPPs. On the other hand, we could also observe that the heat flux for $\mu=0.5 \mathrm{eV}$ is always higher than that for $\mu=0.1 \mathrm{eV}$, while both of them achieve the maximum value at $d_{12}=55 \mathrm{~nm}$. It shows that the coupling role of photons and $\mathrm{Si} /$ Graphene SPPs is absent for $\mu=0.1 \mathrm{eV}$, resulting in that the modulation effect is not significant at this time. The detailed analysis will be performed hereafter. The dash-dotted lines show us the modulation of $T_{2}^{*}$ with $\mu$ and $d_{12}$, which is different for distinct cases of fixed $\mu$ or $d_{12}$. Interestingly, as shown in Fig. 3(a), a minimum value of equilibrium temperature $T_{2}^{*}$ is obtained at around $\mu=0.2 \mathrm{eV}$ when $d_{12}$ keeps unchanged.

To understand the nature of modulation functions with $\mu$ or $d_{12}$, the spectral heat fluxes $\Phi(\omega)$ with a series of $\mu$ and $d_{12}$ are plotted in Fig. 4(a) and 4(b) individually. In both Fig. 4(a) and 4(b), all the spectra exhibit a quasi-monochrome peak with varying degrees of amplitude, which is at frequency $\omega=2.76 \times 10^{14} \mathrm{rad} / \mathrm{s}$. This spectral flux peak was well investigated and understood as the SPPs coupling between two metal or doped semiconductor (doped Silicon for one) plates ${ }^{[4]]}$ However, for low chemical potential $(\mu=0.1 \mathrm{eV})$, a lower secondary peak is observed at around $1.2 \times 10^{14} \mathrm{rad} / \mathrm{s}$, which is
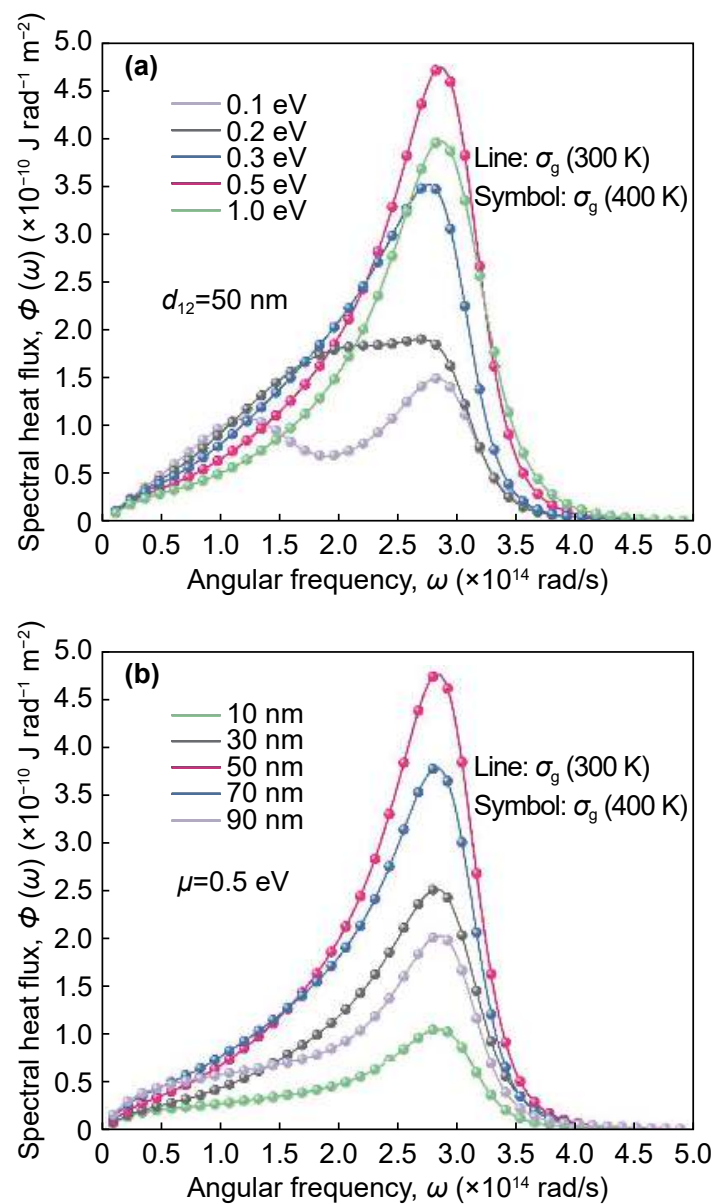

Fig. 4 Near-field spectral radiative heat fluxes $\Phi(\omega)$ between such three-slab configuration at (a) dissimilar graphene chemical potentials $\mu$ as $d_{12}=50 \mathrm{~nm}$, and (b) dissimilar separation distances $d_{12}$ as $\mu=0.5 \mathrm{eV}$. The lines and dots shown in figures correspond to the results obtained from different temperatures $300 \mathrm{~K}$ and $400 \mathrm{~K}$. from the excitation of graphene SPPs. ${ }^{[4]}$ As the chemical potential increases, the graphene SPPs modes blueshift towards higher frequency. Accordingly, the resonant interplay between Graphene and Si SPPs modes starts to take place, leading to resonant role peaks in the vicinity of $\mu=0.5 \mathrm{eV}$. Yet, as the modes move onward with rising $\mu$, the coupling effect of Graphene and Si SPPs dissipates due to gradually inconsistent resonant frequencies. Meanwhile, for the influence of $d_{12}$ on spectral heat flux, there exists a greatly enhanced one at around $d_{12}=50 \mathrm{~nm}$, which is consistent with the results shown in Fig. 4(b). This is because that, as $d_{12}$ changes, the resonance coupling between graphene sheet and emitter or receiver will be varied. The total heat flux is a consequence of both coupling effects and the temperature of graphene sheet. The present results indicate that the symmetric geometry with about $d_{12}=50 \mathrm{~nm}$ could excite strongest coupling effects between emitter, graphene sheet, and receiver. In addition, by fixing other parameters unchanged, we also present separately the corresponding results by taking conductivity of graphene sheet $\sigma_{\mathrm{g}}$ at $300 \mathrm{~K}$ and $400 \mathrm{~K}$. The identical results confirm that the effect of temperature dependent graphene conductivity on the total heat flux is negligible at current case.

In order to describe how sensitive the change of modulation factor is to the increment of chemical potential $\mu$ or separation distance $d_{12}$, we introduce a quantity named sensitivity factor $\mathcal{S}$, which is defined as $\mathcal{S}_{i}=\partial \Omega / \partial \Delta_{i}$ $(i \in\{1,2\})$. It is essentially the slope of modulation factor as a function of $\Delta_{i}$, where $\Delta_{i}\left(\Delta_{1}=\Delta d_{12} / d^{*}, \Delta_{2}=\Delta \mu / \mu^{*}, d^{*}=100\right.$ $\mathrm{nm}$ and $\left.\mu^{*}=1 \mathrm{eV}\right)$ is a new dimensionless number indicating the relative amplitude of variation. As shown in Fig. 5(a), at $\mu=0.5 \mathrm{eV}$ when $\Delta_{1}$ increases from 0.1 to 0.9 , the sensitivity factor $\mathcal{S}_{1}$ first increases to the maximum 5.7 at $\Delta_{1}=0.4$, then decreases rapidly to 0 at $\Delta_{1}=0.6$, where the total heat flux $\Phi$ reaches the maximum. As $\Delta_{1}$ is beyond $0.6, \mathcal{S}_{1}$ becomes negative due to the continuous decrease of $\Phi$ with the increase of $\Delta_{1}$. And then it reaches the minimum value of -4.7 at around $0.8 \mathrm{eV}$. Soon afterwards, $\mathcal{S}_{1}$ monotonically increases to -4 at 0.9 . As for $\mu=0.1 \mathrm{eV}$, the general trend of $\mathcal{S}_{1}$ is gentle, which means that the modulation effect of $d_{12}$ on $\mu=0.1 \mathrm{eV}$ is insensitive. In Fig. 5(b), $\mathcal{S}_{2}$ shows us that the amplification of $\Phi$ with increasing $\Delta_{2}$ is far more sensitive than the abatement of that when $d_{12}$ is constant.

In order to possibly achieve larger thermal modulation effects, we now consider a combination of graphene chemical potential $\mu$ and separation distance $d_{12}$ variation. By arranging different sets of $\mu$ and $d_{12}$ within the domain of $\left(\mu, d_{12}\right)=[0,1] \times[10,90]$, the normalized radiative heat flux $\Omega$ of two-parameter thermal modulator and the equilibrium temperature $T_{2}^{*}$ of intermediate graphene sheet are plotted in Fig. 6(a) and 6(b), respectively. The contour color represents the amplitude of $\Omega$. The results show that largest radiative flux is obtained at $\mu=0.55 \mathrm{eV}, d_{12}=55 \mathrm{~nm}$ under which the coupling of $\mathrm{Si} / \mathrm{Graphene} \mathrm{SPPs}$ is strongest due to perfect 

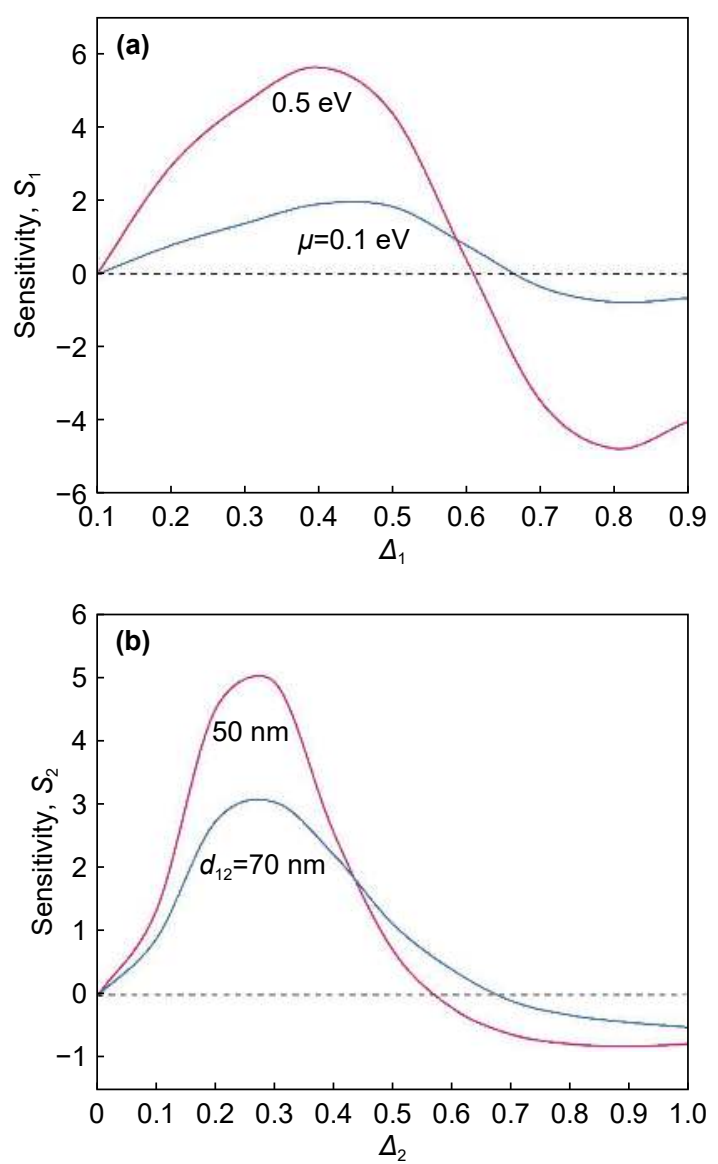

Fig. 5 Radiative heat sensitivity factor $\mathcal{S}_{i}$ as a function of (a) dimensionless separation distance $\Delta_{1}$ when $\mu=0.1$ or $0.5 \mathrm{eV}$ and (b) dimensionless chemical potential $\Delta_{2}$ when $d_{12}=50$ or $70 \mathrm{~nm}$. The dashed lines indicate $\mathcal{S}_{i}=0$.

matching of resonance frequency between doped $\mathrm{Si}$ and Graphene. And the maximum normalized heat flux $\Omega_{\max }\left(\mu, d_{12}\right)$ is 2.64 , which corresponds to the total heat flux of $70 \mathrm{~kW} / \mathrm{m}^{2}$. The minimum value of $\Omega$ reached at $\mu=0 \mathrm{eV}$, $d_{12}=10 \mathrm{~nm}$ is equal to 0.38 due to the resonance frequency mismatch of Si/Graphene SPPs, which has only heat flux of $10 \mathrm{~kW} / \mathrm{m}^{2}$. As a result, the total heat flux can be adjusted from 10 to $70 \mathrm{~kW} / \mathrm{m}^{2}$. Obviously, the two-parameter modulator provides more flexibility to modulate the total heat flux. In contour plot of equilibrium temperature $T_{2}^{*}$, the contour curve is convex towards high temperature as mentioned before with $d_{12}$ beyond $50 \mathrm{~nm}$ and $\mu$ around $0.2 \mathrm{eV}$. However, the contour curve is convex towards low temperature if $d_{12}$ is below $50 \mathrm{~nm}$.

Thermal switching of heat flow belongs to one of major functionalities of thermal modulator, which is also investigated in proposed thermal modulator. The maximum $\Phi_{\max }=\Omega_{\max } \Phi_{0}$ and minimum $\Phi_{\min }=\Omega_{\min } \Phi_{0}$ total radiative flux of modulator are respectively treated to be the "on" or "off" mode of thermal switch to allow or forbid the heat flow to pass. To quantitatively judge how effectively our modulator could switch the heat flow, a new dimensionless factor named switching factor is defined as $\Psi=1-\Omega_{\min } / \Omega_{\max }$ to characterize the performance. According to the results shown in Fig. 6(a) and the definition of switching factor, the overall switching factor is $\Psi=0.85$. Fig. 7 presents $\Psi$ at different chemical potential $\mu$ from 0 to $1 \mathrm{eV}$ or separation distance $d_{12}$ from 10 to $90 \mathrm{~nm}$. As can be observed, it will have a larger
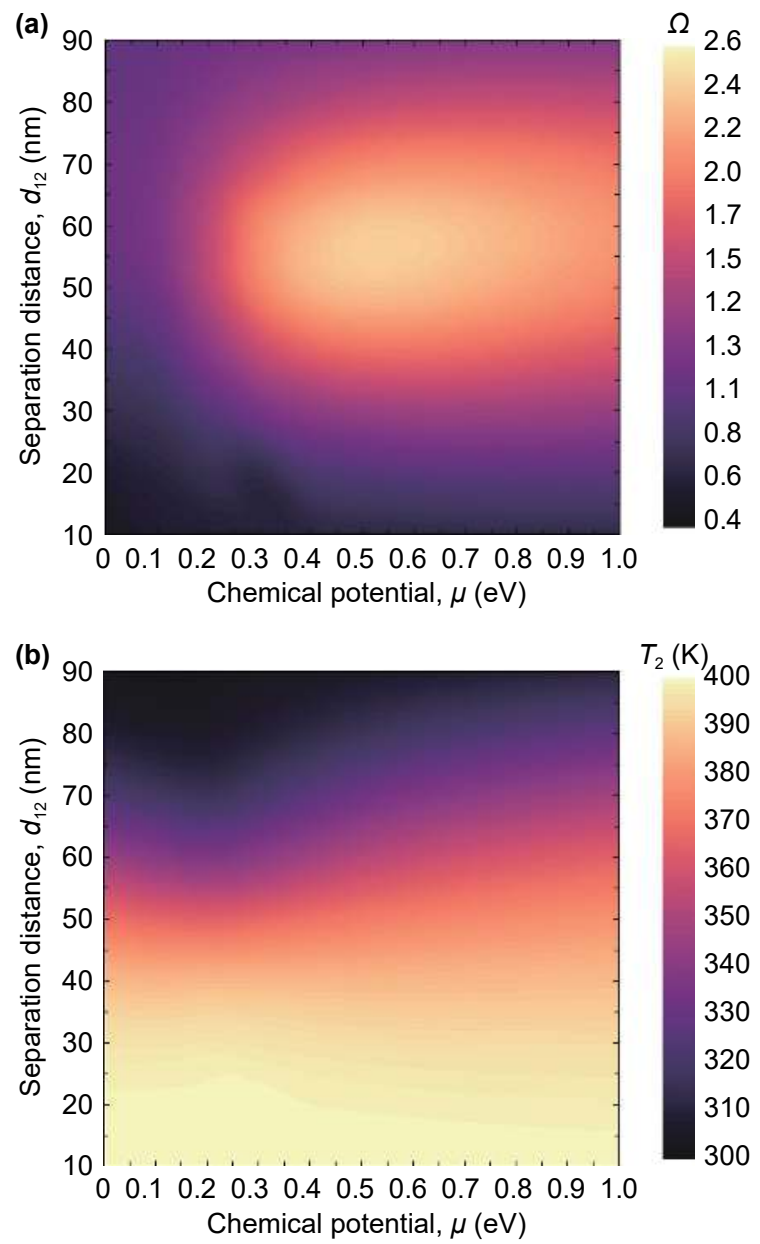

Fig. 6 Normalized radiative heat flux $\Omega$ of three-slab configuration and (b) equilibrium temperatures $T_{2}^{*}$ of intermediate graphene sheet as a function of different sets of graphene chemical potential $\mu$ and separation distance $d_{12}$.

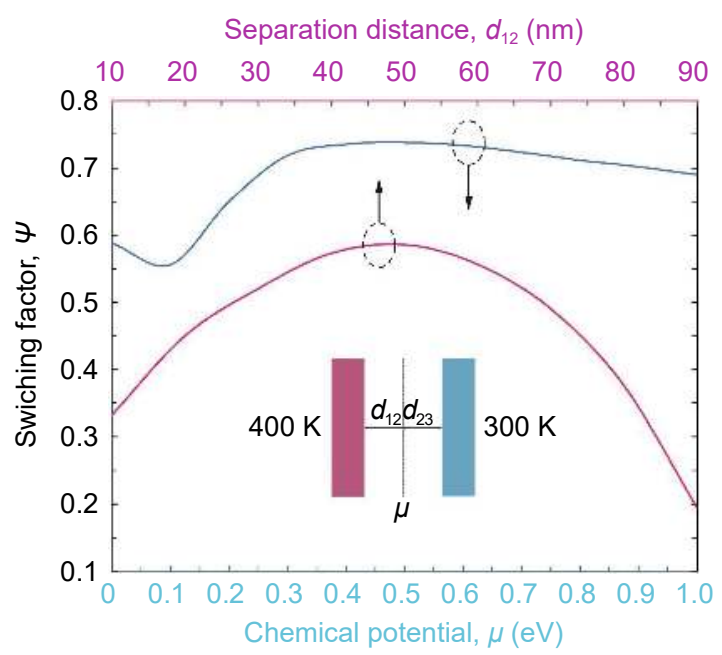

Fig. 7 Radiative thermal switching factor $\Psi$ of such three-slab configuration as a function of graphene chemical potential $\mu$ and separation distance $d_{12}$. 
switching factor with graphene chemical potential fixed than that with fixed separation distance. This is because the heat flux modulation is more sensitive to separation distance change than graphene chemical potential, as illustrated in Fig. 3. The maximum switching factor at around $d_{12}=50 \mathrm{~nm}$ is consistent with previous results because it represents the strongest resonance coupling between there three slabs. In addition, we also notice that the switching factor barely changes with fixed $\mu>0.3 \mathrm{eV}$. This could be explained as that the resonance coupling is weak with $\mu<0.3 \mathrm{eV}$ due to the mismatch of resonance frequency between graphene and $\mathrm{Si}$ SPPs, thus the heat flux modulation range is limited.

\section{Conclusions}

In present work, we have proposed a two-parameter mediated near-field radiative thermal modulator made of Si-graphene-Si configuration, to modulate the total heat flux by photon transport across the three-body system. The graphene sheet as intermediate slab is the main manipulation tool, which is applied to modulate the photon tunneling accurately and speedily with the regulatable chemical potential and separation distance between graphene and emitter. By using a slice of parameter combination, thermal modulation and switch are well realized by this modulator, which obtain a normalized radiative heat flux $\Omega$ in range of $0.38-2.64$ and a switching factor $\Psi=0.85$. The underlying mechanism of tunability is attributed to the coupling effect of graphene and Si surface modes, which gives rise to varied near-field radiative thermal flux with different chemical potentials and separation distances of graphene. By introducing novel 2-D material, near-field thermal transport between polar or hyperbolic materials can be well regulated in the future. This work will shed light on the understanding and design of 2-D material-based near-field thermal devices for active thermal management.

\section{Acknowledgements}

This research was supported by National Natural Science Foundation of China under grant number of 51806045 and Science, and Technology and Innovation Commission of Shenzhen Municipality under grant number of 201771343 and KQJSCX20170329111827540.

\section{Supporting information}

Not applicable

\section{Conflict of interest}

There are no conflicts to declare.

\section{References}

[1] J. Carlos Cuevas and F. J. Garcia-Vidal, ACS Photonics, 2018, 5, 3896-3915.

[2] Y. Tian, A. Ghanekar, M. Ricci, M. Hyde, O. Gregory and Y. Zheng, Mater., 2018, 11, 862

[3] K. Joulain, J. P. Mulet, F. Marquier, R. Carminati and J. J. Greffet,
Surf. Sci. Rep., 2005, 57, 59-112.

[4] Y. Zhang, H. L. Yi and H. P. Tan, ACS Photonics, 2018, 5, 3739-3747.

[5] S. Basu, B. J. Lee and Z. M. Zhang, J. Heat Trans., 2010, 132, $1-8$.

[6] S. Basu, Z. M. Zhang and C. J. Fu, Int. J. Energ. Res., 2009, 33, 1203-1232.

[7] S. Shen, A. Narayanaswamy and G. Chen, Nano Lett., 2009, 9, 2909-2913.

[8] K. Kim, B. Song, V. Fernandez-Hurtado, W. Lee, W. Jeong, L. Cui, D. Thompson, J. Feist, M. T. H. Reid, F. J. Garcia-Vidal, J. C. Cuevas, E. Meyhofer and P. Reddy, Nature, 2015, 528, 387-391.

[9] Y. Yang and L. Wang, Phys. Rev. Lett., 2016, 117, 044301.

[10] Y. Yang, P. Sabbaghi and L. Wang, Int. J. Heat Mass Tran., 2017, 108, 851-859.

[11] S. S. Anandan and V. Ramalingam, Therm. Sci., 2008, 12, 5-26.

[12] P. Ben-Abdallah and S. A. Biehs, Phys. Rev. Lett., 2014, 112, .

[13] K. Joulain, Y. Ezzahri, J. Drevillon and P. Ben-Abdallah, Appl. Phys. Lett., 2015, 106, 133505.

[14] L. P. Wang and Z. M. Zhang, Nanosc. Microsc. Therm., 2013, 17, 337-348.

[15] Y. Yang, S. Basu and L. Wang, Appl. Phys. Lett., 2013, 103, 163101.

[16] K. Ito, K. Nishikawa, H. Iizuka and H. Toshiyoshi, Appl. Phys. Lett., 2014, 105, 253503.

[17] S. Basu and M. Francoeur, Appl. Phys. Lett., 2011, 98, .

[18] A. Ghanekar, J. Ji and Y. Zheng, Appl. Phys. Lett., 2016, 109, 123106.

[19] K. Ito, K. Nishikawa and H. Iizuka, Appl. Phys. Lett., 2016, 108, 053507.

[20] Y. Yang, S. Basu and L. Wang, J. Quant. Spectrosc. Ra., 2015, 158, 69-77.

[21] W. Gu, G. H. Tang and W. Q. Tao, Int. J. Heat Mass Tran., 2015, 82, 429-434

[22] M. J. He, H. Qi, Y. Li, Y. T. Ren, W. H. Cai and L. M. Ruan, Int. J. Heat Mass Tran., 2019, 137, 12-19.

[23] R. Messina, M. Antezza and P. Ben-Abdallah, Phys. Rev. Lett., 2012, 109, 244302.

[24] Y. H. Kan, C. Y. Zhao and Z. M. Zhang, Phys. Rev. B, 2019, 99, 035433.

[25] Z. H. Zheng and Y. M. Xuan, Nanosc. Microsc.Therm., 2011, 15, 237-251.

[26] P. J. van Zwol, K. Joulain, P. Ben-Abdallah and J. Chevrier, Phys. Rev. $B, 2011, \mathbf{8 4}, 161413$

[27] P. J. van Zwol, L. Ranno and J. Chevrier, Phys. Rev. Lett., 2012, 108, 234301.

[28] D. Li and R. B. Kaner, Science, 2008, 320, 1170-1171.

[29] A. N. Grigorenko, M. Polini and K. S. Novoselov, Nat. Photonics, 2012, 6, 749-758.

[30] O. Ilic, M. Jablan, J. D. Joannopoulos, I. Celanovic, H. Buljan and M. Soljacic, Phys. Rev. B, 2012, 85, 155422.

[31] Z. Zheng, X. Liu, A. Wang and Y. Xuan, Int. J. Heat Mass Tran., 2017, 109, 63-72.

[32] J. D. Renteria, D. L. Nika and A. A. Balandin, Appl. Sci.-Basel, 2014, 4, 525-547.

[33] S. Ghosh, I. Calizo, D. Teweldebrhan, E. P. Pokatilov, D. L. Nika, A. A. Balandin, W. Bao, F. Miao and C. N. Lau, Appl. Phys. Lett., 2008, 92, 151911.

[34] M. Lim, S. S. Lee and B. J. Lee, Opt. Express, 2013, 21, 22173-22185

[35] Y. Yang and L. Wang, J. Quant. Spectrosc. Ra., 2017, 197, 68-75. 
[36] A. Fiorino, D. Thompson, L. Zhu, B. Song, P. Reddy and E. Meyhofer, Nano Lett., 2018, 18, 3711-3715.

[37] E. Rousseau, A. Siria, G. Jourdan, S. Volz, F. Comin, J. Chevrier and J. J. Greffet, Nat. Photonics, 2009, 3, 514-517.

[38] H. Wang, Y. Yang and L. P. Wang, J Optics-Uk, 2015, 17, .

[39] G. S. Deng, X. L. Song, S. A. Dereshgi, H. Q. Xu and K. Aydin, Opt. Express, 2019, 27, 23576-23584.

[40] X. Wu, C. Fu and Z. Zhang, J. Photon. Energy, 2019, 9, 032702.

[41] P. A. Huidobro, M. Kraft, S. A. Maier and J. B. Pendry, Acs Nano, 2016, 10, 5499-5506.

[42] Y. Zhang, C. H. Wang, H. L. Yi and H. P. Tan, J. Quant. Spectrosc. Ra., 2018, 221, 138-146.

[43] D. Polder and M. Van Hove, Phys. Rev. B , 1971, 4, 3303-3314.
[44] P. Ben-Abdallah, S. A. Biehs and K. Joulain, Phys. Rev. Lett., 2011, 107, 114301.

[45] R. Messina, P. Ben-Abdallah, B. Guizal, M. Antezza and S. A. Biehs, Phys. Rev. B, 2016, 94, 104301.

[46] M. Lim, S. S. Lee and B. J. Lee, Phys. Rev. B, 2015, 91, 195136.

[47] L. Ju, B. S. Geng, J. Horng, C. Girit, M. Martin, Z. Hao, H. A. Bechtel, X. G. Liang, A. Zettl, Y. R. Shen and F. Wang, Nat. Nanotechnol., 2011, 6, 630-634.

Publisher's Note: Engineered Science Publisher remains neutral with regard to jurisdictional claims in published maps and institutional affiliations. 